\title{
LETTER
}

\section{Forecasting phenology: from species variability to community patterns}

Jeffrey M. Diez, ${ }^{1 *}$ Inés Ibáñez, ${ }^{1}$ Abraham J. Miller-Rushing, ${ }^{2}$ Susan J. Mazer, ${ }^{3}$ Theresa M. Crimmins, ${ }^{4,5}$ Michael A. Crimmins, ${ }^{6}$ C. David Bertelsen ${ }^{7,8}$ and David W. Inouye $e^{9,10}$

\begin{abstract}
Shifts in species' phenology in response to climate change have wide-ranging consequences for ecological systems. However, significant variability in species' responses, together with limited data, frustrates efforts to forecast the consequences of ongoing phenological changes. Herein, we use a case study of three North American plant communities to explore the implications of variability across levels of organisation (within and among species, and among communities) for forecasting responses to climate change. We show how despite significant variation among species in sensitivities to climate, comparable patterns emerge at the community level once regional climate drivers are accounted for. However, communities differ with respect to projected patterns of divergence and overlap among their species' phenological distributions in response to climate change. These analyses and a review of hypotheses suggest how explicit consideration of spatial scale and levels of biological organisation may help to understand and forecast phenological responses to climate change.
\end{abstract}

\section{Keywords}

Climate change, community, Concord, Gothic, phenology, scaling, Tucson, variation.

Ecology Letters (2012) 15: 545-553

\section{INTRODUCTION}

The timing of phenological events influences a wide range of ecological processes, including species' demography (Miller-Rushing et al. 2010), species interactions (Hegland et al. 2009) and ecosystem functions such as carbon cycling (Richardson et al. 2010). Phenological events such as leaf bud burst and flowering, insect emergence and bird migration, are strongly influenced by climate (Root et al. 2005; Parmesan 2006; Rosenzweig et al. 2008). This climate forcing, along with the availability of some long-term datasets, has led to the recognition that phenological change is one of the early indicators that species are responding to changing climate (Zhou et al. 1995; Sparks \& Yates 1997; Menzel \& Fabian 1999). Recent reviews have shown that spring phenological events are changing at an average of 2.3 days per decade and more than 2.5 days per degree Celsius for many species (Menzel et al. 2006a). As a result, there is now an urgency to translate a basic understanding of phenology into forecasts of how phenology will change given continued climate change, and to predict the ecological consequences of these changes.

Although spring as a whole is arriving earlier and the onset of autumn is generally occurring later (Menzel et al. 2006a), there appears to be significant taxonomic, spatial, and temporal variability in the magnitude of this change (Fitter \& Fitter 2002; Gordo \& Sanz 2009;
Primack et al. 2009). Species may show different responses in different parts of their range (Schwartz \& Hanes 2009), different species within the same community may show distinct responses (Cleland et al. 2006; Miller-Rushing \& Primack 2008; Crimmins et al. 2010), and different communities may vary in the direction and strength of responses to climate (Aldridge et al. 2011). Although most studies have shown an overwhelming importance of temperature in shaping phenology, additional environmental variables such as precipitation may be important in some communities (Crimmins et al. 2008, 2010).

This variability has important ecological and evolutionary consequences and can complicate efforts to forecast responses to ongoing climate change (Ibanez et al. 2010; Pau et al. 2011). Within species, variation in phenological responses among individuals or populations may facilitate adaptation to changing conditions and buffer the effects of climate change on the species. Similarly within communities, variation in responses among species will determine community-level patterns of phenology and resilience of species networks to changing climate (Memmott et al. 2007; Kaiser-Bunbury et al. 2010). For example, differences among species in how flowering responds to changing climate will determine the duration of the flowering season and patterns of co-flowering among species, which have important implications for interactions with pollinators and seed predators. If early flowering species respond more strongly to changes in climate

\footnotetext{
${ }^{1}$ School of Natural Resources and the Environment, University of Michigan, Ann Arbor, MI 48109, USA

${ }^{2}$ National Park Service, Schoodic Education and Research Center and Acadia National Park, Winter Harbor, ME 04693, USA

${ }^{3}$ Ecology, Evolution and Marine Biology, University of California, Santa Barbara, Santa Barbara, CA 93106, USA

${ }^{4}$ Office of Arid Lands Studies, University of Arizona, Tucson, AZ 85721, USA

${ }^{5}$ USA National Phenology Network, Tucson, AZ 85721, USA

${ }^{6}$ Department of Soil, Water and Environmental Science, University of Arizona,

PO Box 210038 Tucson, AZ 85721-0038, USA
}

\author{
${ }^{7}$ Herbarium, University of Arizona, PO Box 210036, University of Arizona, \\ Tucson, AZ 85721-0036, USA \\ ${ }^{8}$ School of Natural Resources and the Environment, University of Arizona, \\ Tucson, AZ 85721, USA \\ ${ }^{9}$ Rocky Mountain Biological Laboratory, PO Box 519, Crested Butte, CO 81224, \\ USA \\ ${ }^{10}$ Department of Biology, University of Maryland, College Park, MD 20742-4415, \\ USA
}

*Correspondence: E-mail: jeffdiez@gmail.com 
than later flowering species, as seen in previous study (Menzel et al. 2006b), then the duration of flowering season would be expected to increase rapidly. Evaluating these scenarios will depend on reliable forecasts of how different species and communities are likely to respond to ongoing climate change.

This challenge of forecasting phenology is therefore inseparable from the challenge of scaling. The physiological mechanisms triggering phenological events operate on individuals and can vary at local spatial scales and according to genotype, but we require predictions of the outcomes for populations, species, communities and ecosystems at landscape and regional spatial scales (Cleland et al. 2007). Forecasting phenology thus requires the coordinated analysis of two dimensions of scale: levels of biological organisation and spatial scale. Between the local mechanisms and regional predictions are layers of processes that contribute to phenological variation within and among these levels (Fig. 1). However, development of forecasts at these different scales is constrained by our poor understanding of phenological variability within and among communities, and lack of quantitative analyses bridging these scales.

Herein, we use three long-term datasets of herbaceous plants' first flowering dates (FFDs) to analyse how phenological responses to climate vary within and among communities and to forecast how these communities may differ in their responses to ongoing climate change. We aimed to quantify variation in phenological responses to climate both within and among communities, and asked the following specific questions: (1) How does temperature interact with other climate drivers to shape phenological responses in communities with different climates, including areas where variables other then temperature are known to be important? (2) How do differences among species in their phenological responses to climate scale up to yield patterns of phenology at the community level, including the duration of the flowering season (as measured by FFD) and numbers of species initiating flowering overtime? Analyses presented thus span the spatial scales and levels of organisation in Fig. 1. Because the data analysed here have both strengths and limitations common to historical data, we end with a discussion of the types of data and analyses needed to better understand and forecast ongoing phenological changes.

\section{METHODS}

\section{Datasets}

We analysed three datasets documenting the FFD of herbaceous plant communities from three bioclimatic regions of North America and spanning different time periods: (1) subalpine Gothic, Colorado, from 1973 to 2009 (Inouye 2008; Miller-Rushing \& Inouye 2009; Aldridge et al. 2011), (2) semi-arid Tucson, Arizona from 1984 to 2008 (Crimmins et al. 2009, 2010) and (3) mesic Concord, Massachusetts from 1851 to1858, 1878, 1888 to 1900 and 2003 to 2006 (MillerRushing \& Primack 2008). These datasets have been previously analysed individually and details of data collection can be found in the references elsewhere. Although the specific data collection protocols differed among sites, all datasets have records of: (1) first observed flower dates for the spring herbaceous community (not including graminoids) and (2) climate data during the years of observation. To examine the dynamics of species that tend to flower concurrently in each community, we restricted analysis to spring-flowering species with at least 10 years of data. Because the Tucson data were collected along an 8-km transect ascending over 1200 metres of elevation, we restricted analyses to observations from the first $3.2 \mathrm{~km}$, spanning $426 \mathrm{~m}$ elevation, to minimise variation due to elevation. This selection process resulted in 58 species analysed from Gothic, 42 from Tucson and 38 from Concord. Aside from excluding some rare species, we believe these species to be a representative sample of these communities. Full species lists for each site can be found in the Supplement.

The best available climate data were used for each site. For Concord, monthly climate data from the Blue Hill Meteorological Observatory (33 km from Concord) were used because the Blue Hill data spanned the historical range of phenology data, whereas the closer Bedford, MA NCDC station (5 km from Concord) only began in 1957. Spring precipitation and temperatures were highly correlated (correlation coefficients of 0.91 and 0.96 respectively) between the two stations over the period of overlap from 1957 to 2009, suggesting the Blue Hill data accurately reflected the annual variation in Concord. For Gothic we used data from the Crested Butte weather station (9.5 km from RMBL, and about 210-m lower). Additional data on the

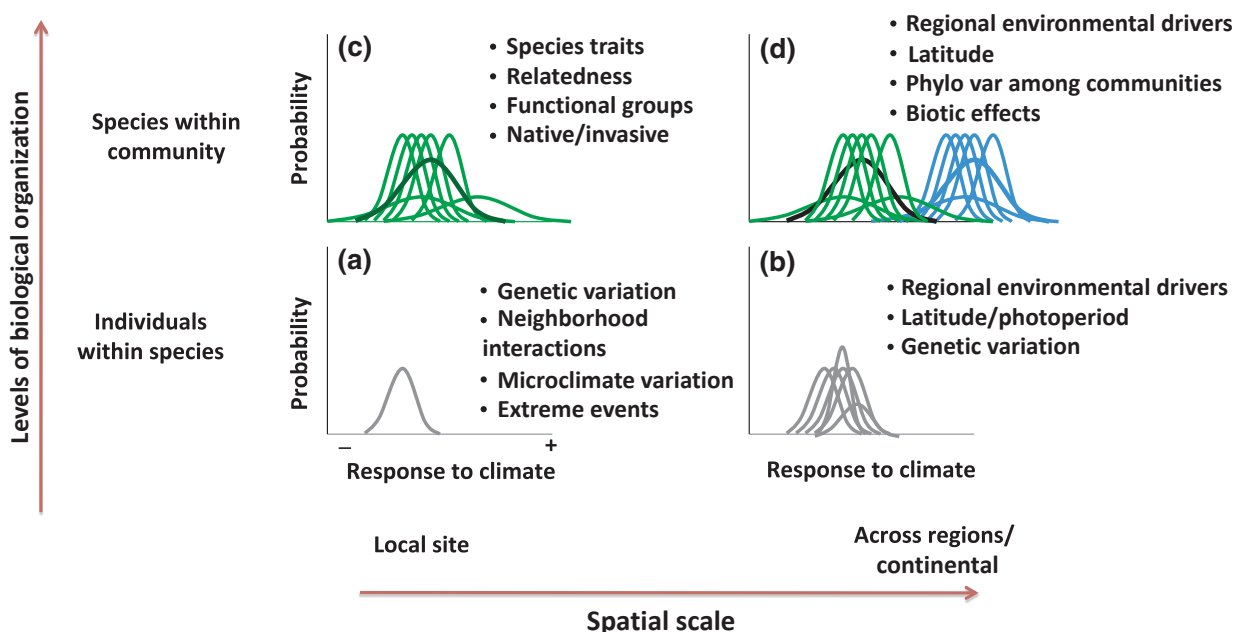

Figure 1 Conceptual diagram showing variability in species' phenological responses to climate at different levels of organisation and spatial scales. Hypotheses proposed to explain variation in phenological responses are relevant at different spatial scales ( $x$-axis) and levels of biological organisation $(y$-axis). Individual curves represent the variation in responses to climate. Phenological responses to climate may vary among individuals within a population (a), among populations across a wider geographic area (b), among species within a particular local community (c) and among communities across a wider geographic scale (d). The dark lines in (c) and (d) represent overall community-level responses. In our analysis of three North American communities, we quantify the variation within and among communities (up to $d$ in figure). 
date of snowmelt were collected at a site located within $1 \mathrm{~km}$ of the study plots (billy barr 2011). For Tucson, monthly temperatures and precipitation were extracted for the study area from the PRISM database (http://www.prism.oregonstate.edu/). The temperature variable used at each site was based on previous study determining the climatic parameter to which spring phenology at the site was most sensitive (see elsewhere references). Mean temperatures between February and April were used for Concord, April and June for Gothic, and October and November for Tucson. Total precipitation values were used for these same time periods for Concord and Tucson, whereas snowmelt dates were used as the additional covariate for Gothic. As with other phenological studies, seasonal climate variables overlap the phenological periods for some species at Concord and Tucson. This yields predictor variables that are affected in part by climate during time periods after the response has occurred. However, the risk of moving the climate window earlier is that the climate becomes too removed from the phenological event. We stick with these time periods because they have proven to be useful predictors of spring phenology in these systems.

\section{Statistical analysis}

Using these data representing three dissimilar communities, our goal was to model how phenological responses to climate varied among species and among communities (corresponding to Fig. 1d). We used four regression models for each community to quantify how species' first flower dates (FFD) changed: (1) overtime, (2) in response to temperature alone, (3) in response to temperature plus additional key climate variables in each region and (4) with interactions between the climatic variables. These additional climate variables, chosen based on previous study in each system, were spring precipitation in Concord, previous late autumn precipitation in Tucson, and dates of winter snow melt in Colorado. The best-supported models, as judged using the model selection criteria DIC (Deviance Information Criterion), were used to forecast likely phenological responses in calendar days given changes from mean observed temperatures up to $4^{\circ}$ higher. Each species' estimated response to temperature was used to predict initiation of flowering in real calendar days across this gradient of increasing temperature.

Regression models were fit in a Bayesian framework, using OpenBUGS (Thomas et al. 2006), to yield posterior probability distributions of regression coefficients that describe changes in FFD of each species overtime, in response to temperature alone, and responses to temperature and the additional driving variable. A Bayesian framework was used to facilitate the interpretation of species' climate sensitivities as probabilities, and to use these relationships to forecast responses as described elsewhere. Noninformative prior distributions were used for all parameters: normal distributions with mean 0 and variance 1000 for regression coefficients and intercepts, and a uniform distribution between 0 and 1000 for the standard deviation parameters. All models were checked for convergence using the Gelman-Rubin statistic and visual inspection after running three chains for 10 000-15000 iterations each after a 5000 iteration burn-in period.

For each community, forecasts were performed across a temperature gradient between the mean observed temperature for the study period and $4{ }^{\circ} \mathrm{C}$ higher. In all communities, this forecasting range could be considered conservative being not far from the observed temperature ranges at each site. In Concord, the forecasting range was from 1.68 to $5.68{ }^{\circ} \mathrm{C}$. The highest mean spring temperature (during the study period) was $4.1{ }^{\circ} \mathrm{C}$ in 1903 and $1.2,3.3,2.8$ and $3.4{ }^{\circ} \mathrm{C}$ from 2003 to 2006 . Forecasted ranges of temperatures were similarly close to the observed range of temperatures in the other communities. In Gothic, the mean April-June temperature over the study period was $8.6{ }^{\circ} \mathrm{C}$. Therefore, forecasts were made over a range from 8.6 to $12.6^{\circ} \mathrm{C}$ and the highest mean spring temperature from the study period, in 1991 , was $12.8^{\circ} \mathrm{C}$. At Tucson, the forecasted range of mean October-November temperature range was from 16.3 to $20.3{ }^{\circ} \mathrm{C}$, not far over the observed high in 2000 of $18.1^{\circ} \mathrm{C}$.

To see how these forecasts interacted with precipitation and snowmelt, predictions for Concord and Tucson were made at five precipitation levels: mean observed precipitation for the study period, highest and lowest observed levels, and the mid-points between mean and extreme values. For Gothic, forecasts were made at five snowmelt dates, ranging from the mean observed snowmelt day (May 20, or day 140 of the year) to 40 days earlier. The earliest observed snowmelt date during the study period was day 112 .

These species-level forecasts were then used to explore the differences in phenological responses among species within communities and the implications of species-specific responses for the distributions of flowering dates in different communities. There are a variety of ways that one might use the species-level forecasts to quantify cumulative changes at the community level. We chose to quantify community-level patterns in two ways that were thought to be ecologically relevant. First, the earliest species' mean flowering date was subtracted from the latest species' mean predicted date to yield a measure of the overall breadth or duration of the community's flowering season. Second, we calculated the number of species with a high likelihood of initiating flowering on each day of the year. This was calculated by summing the number of species on each day whose posterior predictions of FFD were within 0.25 probability of the mean prediction. Summed across all species in a community, this analysis yielded a measure of 'community flowering intensity' that was useful for comparing how the whole community's distribution of flowering times was predicted to change with changes in temperature.

\section{RESULTS}

Species' phenological trends overtime were vastly different among communities (Fig. 2a). The Concord community showed considerably smaller magnitude changes overtime than the other communities, likely due to the long-time frame covered by this dataset (surveys conducted from 1852 to 1858 by H. D. Thoreau, 1888 to 1902 by A. Hosmer and more recently from 2004 to 2006 by A. Miller-Rushing and R. Primack). The other datasets included phenological records from only the latter portion of the 20th century and exhibited greater phenological change overtime. The semi-arid community at Tucson showed significantly more variability than Colorado, in spite of the greater number of species analysed at the latter.

The communities also showed significantly different responses to temperature in models simply relating phenology to temperature (Fig. 2b). The sub-alpine Gothic community showed the strongest response towards earlier phenology with higher temperature; the Concord community exhibited weaker responses, but in the same direction. However, there was also considerable variation among species within each community. The semi-arid Tucson community showed a mean positive response across the whole community (later flowering in warmer years), but species were highly variable and most 
(a)

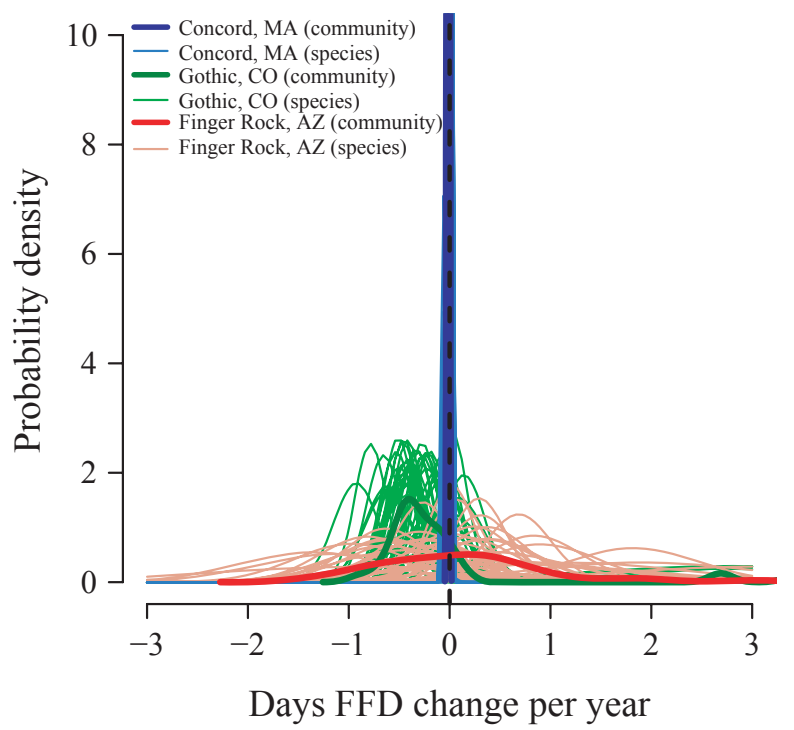

(b)

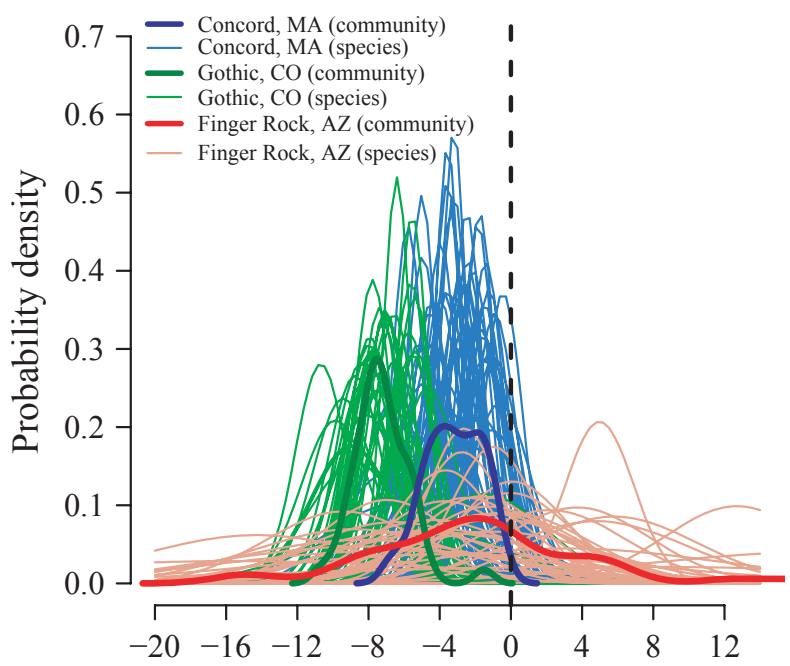

Days FFD change per degree $\mathrm{C}$

(c)

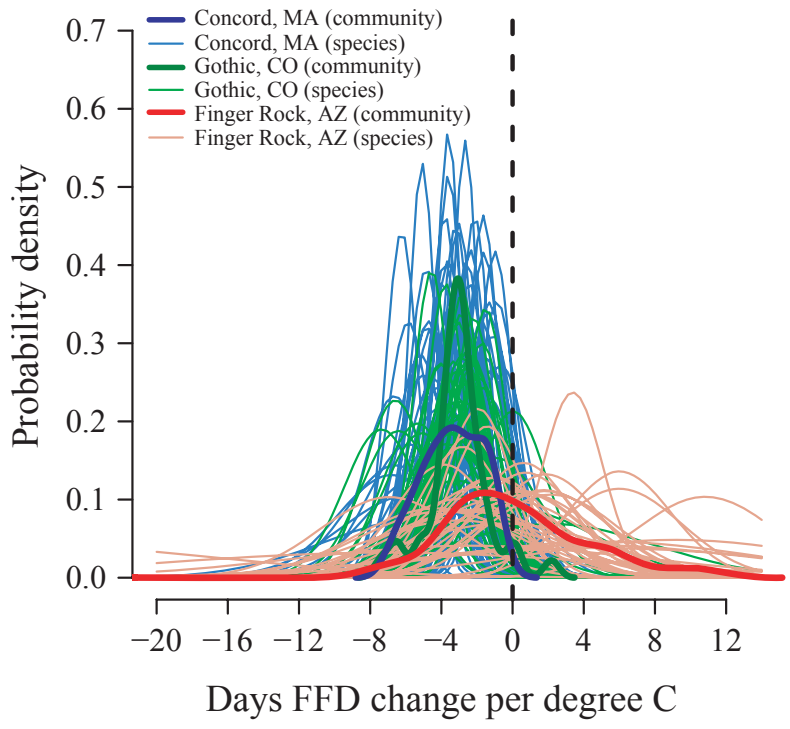

responses overlapped zero suggesting no strong, consistent effect of temperature (Fig. 2b). Thus, there was considerable variation both among species within each community and among communities.

When additional key variables were added to the model at each site, the estimated responses to temperature became much more similar among sites and most of the variability was among species (Fig. 2c). In the sub-alpine Gothic community, snowmelt date was an important driver of phenological events, and the effect of temperature diminished once snowmelt was included in the model. In Tucson, inclusion of precipitation as an explanatory variable caused the effect of temperature to become more prominent and negative (i.e. FFD became earlier with higher temperature). In Concord, adding precipitation to the model did not significantly alter the effect of temperature on flowering date, suggesting a primary role for temperature in this system. Model selection via DIC was consistent with these patterns. The model with lowest DIC (and therefore bestsupported model) for Tucson included the temperature $\times$ precipitation interaction. These interactions varied across species, but eleven species had significantly positive interaction terms $(95 \%$ credible interval did not overlap zero), suggesting stronger temperature effects on FFD when precipitation is higher. For Gothic, the best-supported model included temperature + snowmelt date, but no interaction, and for Concord the model with temperature + precipitation and the interaction model were indistinguishable (full table of DIC values in Supplement). The observed relationships were not qualitatively different across the five levels of precipitation used, so all results are given for the average precipitation values.

Forecasts of expected responses to ongoing climate change showed several differences among communities. The three communities each exhibited high amounts of interspecific variation in the dates of phenological events and responsiveness of these dates to climate (Fig. 3). The overall breadth of FFD in each community, measured as the time from the first species' mean FFD to the last species' mean, differed among the three communities. Tucson had much greater variation among species in the dates of first flowering than did Gothic and Concord (the 'breadth' calculation described in the Methods), and this breadth was also expected to change the most with rising temperatures (Fig. 3). In Concord the breadth changed from 86 days at mean temperatures to 123 days at the highest temperature, while in Gothic breadth changed from 59 to 101 days, and in Tucson from 171 to 234 days. These represent increases of 37,42 and 62 days in Concord, Gothic and Tucson respectively. These changes are also seen in Fig. 4 as the breadths of the start and end points in each community. Unlike the quantitative breadths calculated above based on the means, the shading in the figure is affected by changes in both the mean and uncertainty of each species' FFD. The breadth of event season could increase solely due to increasing uncertainty of species' dates, but in this case the breadth of the means was also increasing. In

Figure 2 Species and community changes overtime and in response to climate. Curves represent the posterior distributions of the regression coefficients describing how species' first flower date (FFD) has changed overtime (a), in response to temperature when only temperature is included in the model (b) and in response to temperature when precipitation is also included in the model (c). Darker, thicker lines represent the empirical distribution of species means (labelled 'community'). The position of the curves on the $x$-axis represents the magnitude and direction of species' responses, where negative values represent earlier flowering overtime (a) or with increased temperature (b \& c). The width of each distribution represents the uncertainty of the relationships. 

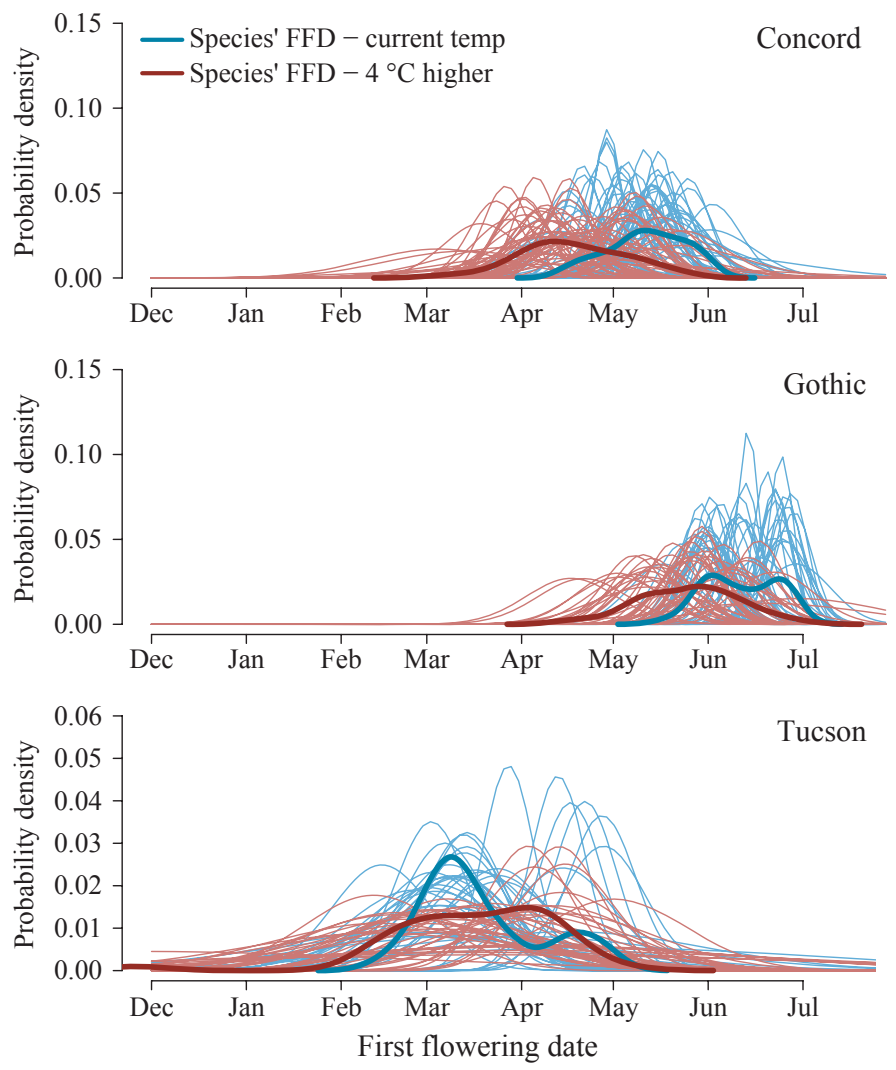

Figure 3 Forecasting expected changes in FFD using relationships with temperature. To explore future overlap and divergence among species, the climate relationships estimated in regression models were used to forecast expected responses in real time, given different climate scenarios. Curves represent posterior predictive probabilities of FFD on given dates for each species. Darker, thicker lines represent the empirical distribution of species means as a way of visualizing overall community-level distributions of dates. Predictions of the FFD for species were made at mean temperatures (blue curves) during the key months (given in methods for each site) and a $4{ }^{\circ} \mathrm{C}$ increase in temperature at each site (red curves).

addition to changing breadth, the distributions and densities of likely flowering dates changed in each community (Fig. 4). Although there is no simple measure to quantify the different patterns of distributions, we discuss below the implications of the apparent differences among communities.

\section{DISCUSSION}

Variability in phenological responses to climate has important implications for species' abilities to adapt to novel environmental conditions and patterns of interactions within communities. Forecasting the outcomes of ongoing climate change will require quantitative approaches for translating species-level variability into expectations for different communities. Our analyses of phenological variability in three plant communities suggest several overall lessons: (1) forecasting future trends in phenology is better performed using relationships with climate than observed trends overtime; (2) there is high variability in phenological responses to climate among species within communities; (3) communities may show similar overall responses to temperature once other regionally important variables are taken into account and (4) despite similar overall responses to
Concord

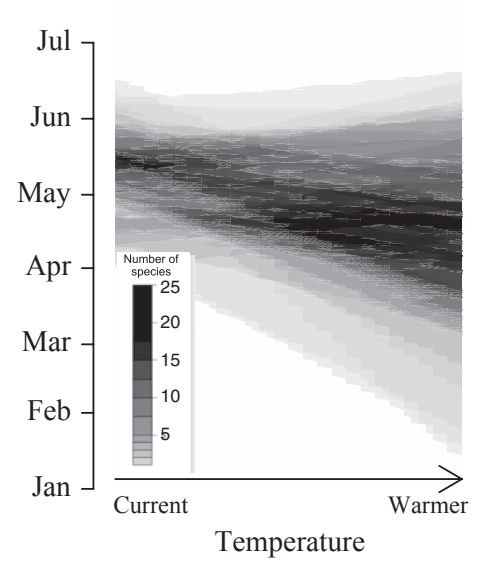

Gothic

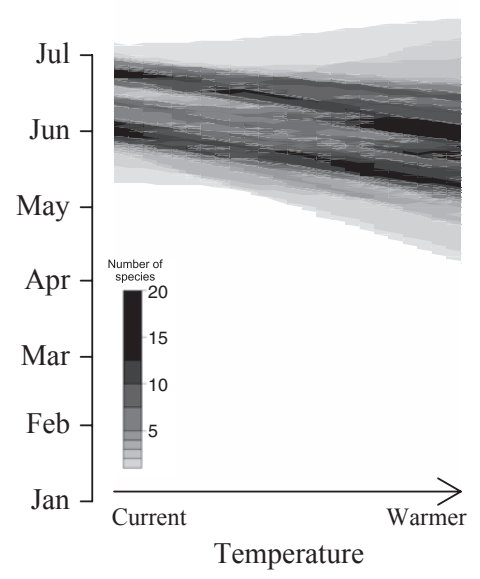

Tucson

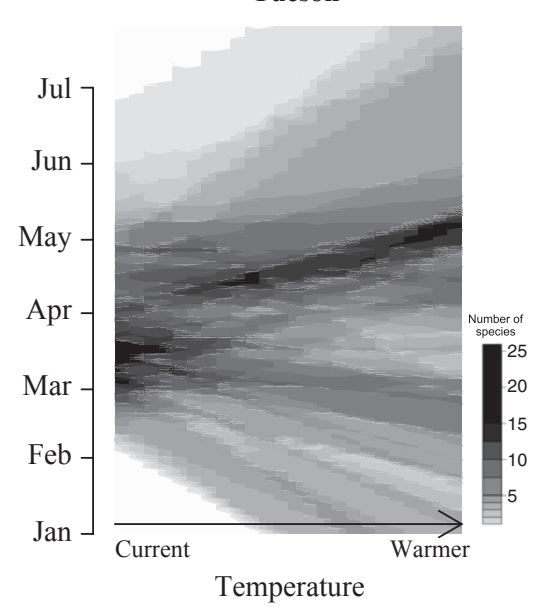

Figure 4 Forecasting community-level changes in distribution of FFD. Shading reflects the number of species with a high likelihood of initiating flowering on each day under different projected temperature increases (where darker shading represents a larger number of species with high likelihood of initiating flowering on that date). These were calculated by summing the number of species on each day whose posterior predictions of FFD were within 0.25 probability of the mean prediction, conditional on a given temperature and at average precipitation. The result is a probabilistic measure of 'community flowering intensity' that represents how the whole community's distribution of flowering times is predicted to shift with changes in temperature. 
temperature, different patterns of community-level phenology may emerge depending on which species are most sensitive to climate.

\section{Phenological changes overtime}

The analyses directly quantifying phenological change overtime illustrate several problems commonly facing efforts to estimate and forecast temporal changes in phenology. We found strong differences among the three communities in their observed trends, with the Concord community exhibiting a comparatively weak trend overtime (Fig. 2a). However, this difference is likely due to the long-time frame covered by this dataset because the timeframe of available data can strongly influence observed trends (Badeck et al. 2004). The other datasets' restriction to the later portion of the 20th century, a period of increased rate of climate change, was a likely cause of the stronger observed trends. In addition to different timeframes of study, changing population sizes can bias observed phenological trends overtime (Miller-Rushing et al. 2008); the larger the population, the more likely that particularly early FFDs will be observed. We do not know the population trends for species in this study and therefore cannot rule out some effects of changes in abundance. Trends in phenology overtime may nonetheless be useful to examine because they reflect all of the factors affecting phenology, including, but not limited to climate. This may be a helpful first step towards assessing the potential for phenological mismatches between species, although the temporal window of analysis must be similar to make comparisons across systems (Bartomeusa et al. 2012).

\section{Species' responses to climate: interactions between drivers}

Species varied considerably in their phenological responses to climate in all three communities (Fig. 2). Because annual temperature variation is greater than the mean trends over the past century, estimated responses to climate are less subject to a dataset's specific timeframe and therefore more comparable across systems than changes overtime. Comparing these three communities' climatic relationships revealed two lessons of general interest to phenological studies. First, the importance of including additional regional climate variables depends on the bioclimatic region. Snowmelt date was an important driver of phenological events in the subalpine Gothic community, and the effect of temperature diminished once snowmelt was included in the model (Fig. 2b, c). This suggests both a direct and indirect role for temperature in this system, and is consistent with mechanisms of how snow cover during the spring is thought to influence plant phenology in subalpine ecosystems (Inouye 2008).

In Tucson, the effect of temperature became more prominent and negative (i.e. FFD became earlier with higher temperature) once precipitation was included, consistent with the important role of precipitation in semi-arid ecosystems (Noy-Meir 1973). Tucson was also the only community for which the model including an interaction between temperature and precipitation was favored in model selection. The positive interactions between temperature and precipitation suggested that these climate variables act synergistically to affect species phenology. Warm temperatures appear to have a greater effect on flowering when suitable moisture conditions exist. Research on model organisms has shown how a combination of endogenous (related to developmental state) and environmentally triggered pathways may be important for triggering phenology (Simpson \&
Dean 2002), suggesting the possibility that precipitation and temperature are affecting phenology both through proximate mechanisms and interactive effects on plant development.

In contrast to Tucson, adding precipitation to the model for the Concord site did not significantly alter the effect of temperature on flowering date, suggesting a primary role for temperature in this system. This minimal effect of precipitation is not surprising given the relatively mesic spring conditions of the northeast deciduous biome. These results highlight the importance of understanding regional variation in key climatic drivers and incorporating them into forecasts of responses to climate change.

After incorporating snowmelt dates and precipitation, the community-level responses to temperature became quite similar across systems (Fig. 2c), suggesting an underlying similarity in how suites of species respond to temperature. This convergence resulted despite considerable variation among species within these sites and variation among sites in taxonomic composition. This convergence suggests the possibility of broad constraints on how temperature affects the biochemical pathways underlying flowering pathways in herbaceous plants (Amasino 2010). Such a pattern also suggests the possibility of finding scales at which predictive relationships between phenological change and climate change can be applied to other communities. Although the responses of unstudied species may not be readily predictable until the sources of among species variation are better understood, overall expectations at the community level may be more likely to follow these general patterns. Further tests in additional communities, as data become available, should help reveal the generality of this pattern.

\section{Forecasting: from species variability to community phenology patterns}

Species' phenological responses to climate were used to forecast changes for each species under a gradient of increasing temperature and to explore community-level patterns of change. One metric of this change at the community level is the breadth of the event season. For plants, phenological events early in the year often exhibit stronger and perhaps more variable responses to temperature than those later in the year (Menzel et al. 2006b). This kind of partitioning of the strength of responses in time would be expected to yield significant divergence among species with further warming. However, in this study we found no obvious temporal partitioning or divergence of responses in these flowering communities (Fig. S1); both early and late flowering spring species exhibited similar responses to a given increase in temperature. Nonetheless, the breadth of species' FFDs (the time from the earliest species' mean FFD to the latest species' mean FFD) was predicted to become wider in all three communities, and the magnitude of these changes differed among communities. In particular, Tucson exhibited greater variability in projected responses among species (Fig. 2) and a larger shift in breadth than Gothic and Concord (Fig. 3). With only one representative community from each biome, we cannot disentangle the effects of climate variation from differences in species pools. Nonetheless, the wide variation and divergence observed in Tucson may be more common in arid environments in which high annual variability in precipitation amounts and timing may drive highphenological variability. Variability in fall precipitation in Tucson has strong effects on the timing of phenological events in the following spring (Crimmins et al. 2008, 2010). Additional factors may also 


\section{Box 1 Hypotheses to explain phenological variation at different levels of organisation}

A number of hypotheses have been proposed to explain variation in phenology. This is not an exhaustive review, but rather shows a representative subset of plant-focused hypotheses to illustrate how they can be organised to help explain phenological variation at different levels of organisation. Full references for literature cited are included as online Supporting Information.

The studies organised in the table below suggest several additional issues of general relevance to phenological research. First, many studies of phenology are based on observational data, making direct causal relationships difficult to confirm (as discussed by Elzinga et al., 2007). When possible, additional efforts should be made to supplement these correlational tests of hypotheses using experimental, manipulative approaches. Second, proximate causes of phenological variation (e.g. phenotypically plastic responses to local environmental conditions or cues) are typically not distinguished from ultimate causes (e.g. adaptive phenological responses inherited from ancestral taxa). In fact, studies of phenological variation often confound genetic (and potentially adaptive) and environmentally induced differences in responses. To the extent that responses are adaptive, relatedness among individuals at different taxonomic levels (genus, family) may help explain phenological variation. This 'phylogenetic' hypothesis can be tested without knowledge of the underlying evolutionary mechanisms, but will be more powerful when linked to an understanding of how specific life history traits affect proximate phenological responses. This confounding of mechanisms can also occur at the community level, as communities typically differ simultaneously in taxonomic composition and environmental drivers. Together, these issues highlight the difficulty of assigning unambiguous causes to observed phenological patterns in the field. Ongoing research aimed at teasing apart these hypotheses should make efforts where possible to minimise these confounding effects. Abbreviations in table: FFD = first flowering date; PFD = peak flowering date; LFD = last flowering date.

\section{Ecological level}

Within individual

Among individuals, within species
Phenological trait

FFD

Date of vegetative budburst and FFD

Among populations

Mean date of vegetative budburst and FFD

Among species
Mean FFD, PFD, LFD, duration, synchrony and/or frequency
Cause of variation [example citation]

Plant age (Van Dijk 2009)

Branch position (axillary vs. terminal; Tapingkae et al. 2007)

Spatial variation in environmental conditions: e.g. cumulative degree days, thawing degree days, vernalisation, soil moisture, drought index, snowmelt; soil temperature (Primack et al. 1980; Wielgolaski 2001; Quinn \& Wetherington 2002; Dunne et al. 2003; Inouye \& Wielgolaski 2003; Inouye 2008; Alizoti et al. 2010; Haggerty \& Galloway 2011)

Resource availability: plant size, availability of stored vegetative resources (Mazer 1987; Dieringer 1991; Ollerton \& Lack 1998; Lacey et al. 2003; Baker et al. 2005; Sola \& Ehrlén 2007; Bustamante \& Burquea 2008; Latta \& Gardner 2009; Haggerty \& Galloway 2011); maternal effects (Lacey et al. 2003); mass of sown seed (Mazer 1987)

Damage: cotyledon or leaf damage (Marquis 1988; Hanley \& Fegan 2007); Timing of cotyledon

damage (Hanley \& Fegan 2007); pathogen infection (Korves \& Bergelson 2003)

Genotype (genes influencing FFD, PFD, response to photoperiod, plant size and other traits genetically correlated with FFD and PFD; Mazer 1987; Lacey et al. 2003; Putterill et al. 2004; Weis \& Kossler 2004; Baker et al. 2005; Burgess et al. 2007; Dijk 2009; Ivey et al. 2009; Latta \& Gardner 2009; Scarcelli \& Kover 2009; Alizoti et al. 2010;

Wilczek et al. 2010)G $\times$ E interactions (Harris et al. 2006; Johnson 2006)

Neighborhood density (Mazer \& Schick 1991)

History of natural selection on response to environmental cues (O’Neil 1997; Aizen 2003; Hall \& Willis 2006; Elzinga et al. 2007; Franks et al. 2007; Sandring et al. 2007; Franks \& Weis 2008; Sandring \& Ågren 2009;

Kawagoe \& Kudoh 2010)

Elevation/aspect (Bertiller et al. 1990; Peterson 1997; Haggerty \& Galloway 2011)

Presence of invasive species (Wilke \& Irwin 2010)

Temperature or vernalisation (Price \& Waser 1998; Cleland et al. 2006; Post et al. 2008)

Photoperiod $\times$ temperature interaction (Heide \& Sønsteby 2007;

Craufurd \& Wheeler 2009; Wilczek et al. 2010)

Mating system (Elle et al. 2010)

Abundance (Miller-Rushing et al. 2008)

Native vs. exotic (Wolkovich \& Cleland 2011)

Duration of flowering (Bawa 2003; Osada et al. 2003)

Photoperiod (Stevenson et al. 2008)

Traits: fleshy vs. non-fleshy fruits (Bolmgren \& Lönnberg 2005); pollination syndrome (Heinrich 1976; Bolmgren et al. 2003; Bolmgren \& Cowan 2008; Du \& Qi 2010); seed mass (Mazer 1989, 1990; Bolmgren \& Cowan 2008; Du \& Qi 2010); plant size (Bolmgren \& Cowan 2008; Du \& Qi 2010)

Phylogenetic relatedness, but underlying cause unknown (Mazer 1990; Lobo et al. 2003; Borchert 2004; Debussche et al. 2004; Brearley et al. 2007; Bolmgren \& Cowan 2008; Davis et al. 2010; Staggemeier et al. 2010) 
Box 1 (continued)

\begin{tabular}{lll}
\hline Ecological level & Phenological trait & Cause of variation [example citation] \\
\hline Among communities & Mean FFD and PFD among taxa, & Disturbance or habitat type (Heinrich 1976) \\
& Ra length or peak of fruit production & Geographic effects: altitude/temperature (Arroyo $e t$ al. 1981; Petanidou $e t$ al. 1995); \\
& latitude (Ting $e t$ al. 2008); aspect (Arroyo $e t$ al. 1981); photoperiod (Borchert 2004) \\
& Seasonal variation in rainfall and temperature in tropical dry forests and rain forests \\
& (Borchert 2004; Boulter $e t$ al. 2006) \\
\hline
\end{tabular}

contribute to high variability in Tucson, including the diversity of elevations and habitats (transects used here spanned $426 \mathrm{~m}$ elevation and traversed desert scrub, riparian scrub and scrub grassland habitats), but more detailed climate data is needed to assess the importance of microclimate. Using first flower dates as the response variable, the warmest sites occupied by each species are in effect sampled every year. The estimates of species' sensitivities to climate (i.e. days of phenological change per degree Celsius, Fig. 2), and the forecasts based on these sensitivities (Figs 3 and 4), should not be affected by differences among microsites.

In addition to the projected increased breadth of the FFD, there are many more complex changes resulting from shifting frequencies of events over the season. The most relevant aspect of change at the community level will depend on the question of interest; however, thereby eluding simple quantitative metrics as calculated for breadth. Events may become more clumped in time or more divergent, each with different implications for species interactions. Divergent patterns among species may interrupt interactions to which species were previously adapted, and convergent timing will create novel interactions (Memmott et al. 2007). In the three communities analysed here, we see interesting differences in projected patterns of overlap and divergence among species (Fig. 4). For example, flowering dates in Concord actually converged towards being more concentrated in one part of the year, despite an overall increase in breadth from earliest to latest species (Fig. 4a). By contrast, the density of flowering times remained more constant in Gothic, and became more diffuse in Tucson (Fig. 4b, c). Each scenario holds different implications for how species interactions might change with changing climate. A recent community-level analysis for the whole flowering season in Gothic found that as the season becomes longer, a mid-season gap in flower abundance begins to develop, emphasizing the importance of looking at the breadth of phenological events at the community level (Aldridge et al. 2011). An additional consequence of earlier flowering dates in Gothic may be a decline in flower abundance due to increased frost damage (Inouye 2008). These differences suggest that there are not necessarily general rules that will apply across communities; however, more comparisons across communities are needed to test this.

\section{Moving forward: confronting the complexity of phenological change across scales}

The need to bridge different scales of phenological responses is widely recognised (Cleland et al. 2007), but remains very difficult due to the complexity of processes linking these scales. Our analyses of three excellent long-term datasets provide novel perspectives on this challenge, but also highlight limitations of historical datasets. We conclude here by outlining a path towards improved multi-scale phenological forecasts that includes testing scale-explicit hypotheses, collecting targeted data and developing theory.

The wide variety of hypotheses that have been proposed to explain different aspects of phenological variability (Forrest \& Miller-Rushing 2010) can be organised by spatial scale and level of organisation (Box 1). As tests of these hypotheses help explain variation observed at different scales, we can identify the primary drivers that will be most useful for forecasting (Clark et al. 2001). To do this, several types of data are needed that are typically not available in historical records. Most significantly, we need data spanning entire phenophases instead of single events such as first flower dates. For example, data on the flowering duration of species in different years would be much more useful than first flower dates for understanding how floral resources for pollinators are likely to change with climate change. Similarly, data on the abundance of flowers would help to extend these analyses to quantitative measures of floral availability. Coupling these quantitative phenological data to demographic outcomes (fecundity, survival and growth) will also extend phenological analyses to understand how changes in phenology, and resulting changes in species interactions, affect fitness, an important currency for understanding lasting impacts of phenological change. Finally, ecological theory may play a critical role in reducing the complexity of these problems into more manageable pieces. It may not be tractable to forecast phenological trends for all species, but there may be aggregated units of organisation to focus on for understanding overall community dynamics. For example, recent study has explored how to organise interaction networks by groups (Allesina \& Pascual 2009) or specific interaction 'modules' (Gilman et al. 2010), which may be particularly relevant to understanding community responses to climate change.

\section{ACKNOWLEDGEMENTS}

We would like to thank three anonymous referees and the editors for extremely helpful comments. We also thank Oscar Gordo, Sean McMahon, Sarah McCarthy-Neumann, Pablo González Moreno, Dan Katz, and the Ibáñez lab group for valuable comments on an early draft of the manuscript, and Richard Primack for his comments on the manuscript and for allowing us to use the phenological observations in Concord, MA. We are also very grateful to George Aldridge for help with the Gothic, CO data. Data collection and analysis were supported by the National Science Foundation (DEB-0238331, DEB 0922080; David Inouye).

\section{AUTHORSHIP}

CDB, DWI, AMR, MAC collected data and TMC managed data; AMR initiated the project; JMD, II, AMR, SJM developed ideas; JMD 
performed analyses and wrote the paper; II contributed to analyses; SJM performed literature review to compile Box 1; SJM, II, AMR, TMC, CDB, DWI, MAC edited the paper.

\section{REFERENCES}

Aldridge, G., Inouye, D.W., Forrest, J., Barr, W.A. \& Miller-Rushing, A.J. (2011). Emergence of a mid-season period of low floral resources in a montane meadow ecosystem associated with climate change. J. Ecol., 99, 905-913.

Allesina, S. \& Pascual, M. (2009). Food web models: a plea for groups. Ecol. Lett., $12,652-662$.

Amasino, R. (2010). Seasonal and developmental timing of flowering. Plant J., 61, 1001-1013.

Badeck, F.W., Bondeau, A., Bottcher, K., Doktor, D., Lucht, W., Schaber, J. et al. (2004). Responses of spring phenology to climate change. New Phytol., 162, 295-309.

Barr, B. (2011). Available at: http://rmbl.info/rockymountainbiolab/con/con_bcc_data.html. Last accessed 5 May 2010.

Bartomeusa, I., Ascher, J.S., Wagner, D., Danforth, B.N., Colla, S., Kornbluth, S. et al. (2012). Climate-associated phenological advances in bee pollinators and bee-pollinated plants. Proc. Nat. Acad. Sci. USA, 108, 20645-20649.

Clark, J.S., Carpenter, S.R., Barber, M., Collins, S., Dobson, A., Foley, J.A. et al. (2001). Ecological forecasts: an emerging imperative. Science, 293, 657-660.

Cleland, E.E., Chiariello, N.R., Loarie, S.R., Mooney, H.A. \& Field, C.B. (2006). Diverse responses of phenology to global changes in a grassland ecosystem. Proc. Nat. Acad. Sci. USA, 103, 13740-13744.

Cleland, E., Chuine, I., Menzel, A., Mooney, H. \& Schwartz, M. (2007). Shifting plant phenology in response to global change. Trends Ecol. Evol., 22, 357365.

Crimmins, T., Crimmins, M., Bertelsen, D. \& Balmat, J. (2008). Relationships between alpha diversity of plant species in bloom and climatic variables across an elevation gradient. Int. J. Biometeorol., 52, 353-366.

Crimmins, T.M., Crimmins, M.A. \& Bertelsen, C.D. (2009). Flowering range changes across an elevation gradient in response to warming summer temperatures. Glob. Change Biol., 15, 1141-1152.

Crimmins, T.M., Crimmins, M.A. \& Bertelsen, C.D. (2010). Complex responses to climate drivers in onset of spring flowering across a semi-arid elevation gradient. J. Ecol., 98, 1042-1051.

Fitter, A. \& Fitter, R. (2002). Rapid changes in flowering time in British plants. Science, 296, 1689-1691.

Forrest, J. \& Miller-Rushing, A.J. (2010). Toward a synthetic understanding of the role of phenology in ecology and evolution. Philosophical Transactions of the Royal Society B-Biological Sciences, 365, 3101-3112.

Gilman, S.E., Urban, M.C., Tewksbury, J., Gilchrist, G.W. \& Holt, R.D. (2010). A framework for community interactions under climate change. Trends Ecol. Evol., 25, 325-331.

Gordo, O. \& Sanz, J.J. (2009). Long-term temporal changes of plant phenology in the Western Mediterranean. Glob. Change Biol., 15, 1930-1948.

Hegland, S.J., Nielsen, A., Lazaro, A., Bjerknes, A.L. \& Totland, O. (2009). How does climate warming affect plant-pollinator interactions? Ecol. Lett., 12, 184 195.

Ibanez, I., Primack, R.B., Miller-Rushing, A.J., Ellwood, E., Higuchi, H., Lee, S.D. et al. (2010). Forecasting phenology under global warming. Phil. Trans. R. Soc. B: Biol. Sci., 365, 3247-3260.

Inouye, D.W. (2008). Effects of climate change on phenology, frost damage, and floral abundance of montane wildflowers. Ecology, 89, 353-362.

Kaiser-Bunbury, C.N., Muff, S., Memmott, J., Müller, C.B. \& Caflisch, A. (2010). The robustness of pollination networks to the loss of species and interactions: a quantitative approach incorporating pollinator behaviour. Ecol. Lett., 13, $442-452$.

Memmott, J., Craze, P.G., Waser, N.M. \& Price, M.V. (2007). Global warming and the disruption of plant-pollinator interactions. Ecol. Lett., 10, 710-717.

Menzel, A. \& Fabian, P. (1999). Growing season extended in Europe. Nature, 397, 659-659.
Menzel, A., Sparks, T., Estrella, N., Koch, E., Aasa, A., Ahas, R. et al. (2006a). European phenological response to climate change matches the warming pattern. Glob. Change Biol., 12, 1969-1976.

Menzel, A., Sparks, T., Estrella, N. \& Roy, D. (2006b). Altered geographic and temporal variability in phenology in response to climate change. Glob. Ecol. Biogeogr., 15, 498-504.

Miller-Rushing, A.J. \& Inouye, D.W. (2009). Variation in the impact of climate change on flowering phenology and abundance: an examination of two pairs of closely related wildflower species. Am. J. Bot., 96, 1821-1829.

Miller-Rushing, A. \& Primack, R. (2008). Global warming and flowering times in Thoreau's Concord: a community perspective. Ecology, 89, 332-341.

Miller-Rushing, A.J., Inouye, D.W. \& Primack, R.B. (2008). How well do first flowering dates measure plant responses to climate change? The effects of population size and sampling frequency. J. Ecol., 96, 1289-1296.

Miller-Rushing, A., Høye, T., Inouye, D. \& Post, E. (2010). The effects of phenological mismatches on demography. Pbil. Trans. R. Soc B: Biol. Sci., 365, 3177.

Noy-Meir, I. (1973). Desert ecosystems: environment and producers. Annu. Rev. Ecol. Syst., 4, 25-51.

Parmesan, C. (2006). Ecological and evolutionary responses to recent climate change. Annu. Rev. Ecol. Evol. Syst., 37, 637-669.

Pau, S., Wolkovich, E.M., Cook, B.I., Davies, T.J., Kraft, N.J.B., Bolmgren, K. et al. (2011). Predicting phenology by integrating ecology, evolution and climate science. Glob. Change Biol., 17, 3633-3643.

Primack, R.B., Ibanez, I., Higuchi, H., Lee, S.D., Miller-Rushing, A.J., Wilson, A.M. et al. (2009). Spatial and interspecific variability in phenological responses to warming temperatures. Biol. Conserv., 142, 2569-2577.

Richardson, A., Andy Black, T., Ciais, P., Delbart, N., Friedl, M., Gobron, N. et al. (2010). Influence of spring and autumn phenological transitions on forest ecosystem productivity. Phil. Trans. R. Soc B: Biol. Sci., 365, 3227.

Root, T.L., MacMynowski, D.P., Mastrandrea, M.D. \& Schneider, S.H. (2005). Human-modified temperatures induce species changes: joint attribution. Proc. Nat. Acad. Sci. USA, 102, 7465-7469.

Rosenzweig, C., Karoly, D., Vicarelli, M., Neofotis, P., Wu, Q.G., Casassa, G. et al. (2008). Attributing physical and biological impacts to anthropogenic climate change. Nature, 453, 353-358.

Schwartz, M.D. \& Hanes, J.M. (2009). Continental-scale phenology: warming and chilling. Int. J. Climatol., 30, 1595-1598.

Simpson, G.G. \& Dean, C. (2002). Arabidopsis, the rosetta stone of flowering time? Science, 296, 285-289.

Sparks, T.H. \& Yates, T.J. (1997). The effect of spring temperature on the appearance dates of British butterflies 1883-1993. Ecography, 20, 368-374.

Thomas, A., O’Hara, R.B., Ligges, U. \& Sturtz, S. (2006). Making BUGS open. $R$ News, 6, 12-17.

Zhou, X.L., Harrington, R., Woiwod, I.P., Perry, J.N., Bale, J.S. \& Clark, S.J. (1995). Effects of temperature on aphid phenology. Glob. Change Biol., 1, 303-313.

\section{SUPPORTING INFORMATION}

Additional Supporting Information may be downloaded via the online version of this article at Wiley Online Library (www.ecologyletters.com).

As a service to our authors and readers, this journal provides supporting information supplied by the authors. Such materials are peer-reviewed and may be re-organised for online delivery, but are not copy edited or typeset. Technical support issues arising from supporting information (other than missing files) should be addressed to the authors.

\section{Editor, Elsa Cleland}

Manuscript received 9 November 2011

First decision made 8 December 2011

Second decision made 9 February 2012

Manuscript accepted 23 February 2012 